\title{
Measurement of Electric-Field Intensities Using Scanning Near-Field Microwave Microscopy
}

\author{
Roman Kantor and I. V. Shvets
}

\begin{abstract}
In this paper, we propose methods for the measurement of electric intensities of a microwave field above the surface of microwave circuits. Using miniaturized coaxial antennas, we measure all spatial components of the induced field above device-under-test. A special position/signal difference method is used to better localize the measured field and increase the spatial resolution of the field mapping. During the scanning process, the antenna is driven at the defined distance above the sample surface according to previously acquired topographic data. For measurement of the tangential-field components parallel to the sample surface, the antenna is tilted by approximately $45^{\circ}$ relative to the sample surface. By its rotation about the vertical axis, various components of the field are measured, and vertical and horizontal electric-field intensities are recalculated. The probes are calibrated in a well-defined field standard and allow good quantitative characterization of the measured field.
\end{abstract}

Index Terms-Coaxial antennas, near-field measurements, scanning near-field microscopy.

\section{INTRODUCTION}

C ONTEMPORARY systems for inspection of microwave circuits are usually based on the measurement of signals at the device ports. In most cases, they are limited to the measurement of the input and output signals. Such measurements are often not sufficient for characterizing the functionality of individual elements and do not give a full description of distribution of circuit signals. As a result, noncontact scanning near-field measurements become an attractive method for testing circuit performance and failure analysis. By analyzing the electric- and magnetic-field distribution above the circuit surface, one can quantitatively evaluate the field sources, charge, and current pattern [1]. Knowledge of the field distribution can also explain the signal coupling between the circuit components and may also be used for the description of the electromagnetic emission and other aspects of electromagnetic compatibility (EMC) of the device-under-test.

\section{Electric-Field ANTENNAS AND Position/Signal DIFFERENCE METHOD}

For acquisition of microwave electric intensities in a nearfield region, short coaxial antennas [1], [2] are commonly used. In some cases, a modification in the probe geometry, such as the use of slit probes [3], [4], small dipole antennas, and open-ended transmission lines [5], [6] can increase signal-matching effi-

Manuscript received November 27, 2002; revised April 24, 2003. This work was supported by the Science Foundation Ireland under Contract 00/PI.1/C042.

The authors are with the Science Foundation Ireland Laboratories, Physics Department, Trinity College, Dublin 2, Ireland.

Digital Object Identifier 10.1109/TMTT.2003.818938

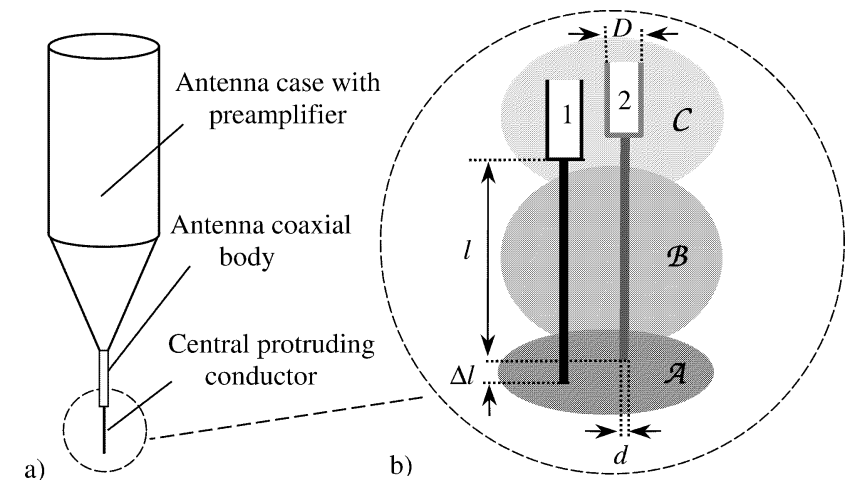

Fig. 1. Electric-field probe and principle of PSD method. (a) Outline. (b) Central protruding conductor. The two positions of the antenna corresponding to different heights above the surface of the device-under-test are indicated in (b) by 1 and 2. Our antennas have a shield with an outer diameter of $D=230 \mu \mathrm{m}$ and a thin relatively long $(l \gg d)$ central protruding conductor: a copper wire of length $l \simeq 0.3-1 \mathrm{~mm}, d=8 \mu \mathrm{m}$.

ciency, change the polarization, or limit the region to which the antenna is sensitive. Due to good sensitivity and well-defined electric properties, we have focused our attention on the miniaturized coaxial antennas-cylindrical short monopoles-where a central conductor protrudes for a defined length from the shielding (see Fig. 1). Due to the axial symmetry, such antennas are sensitive to the component of the electric-field intensity parallel to that axis. The external field is commonly assumed to be homogeneous, thus resulting in a single sensitivity coefficient, which is the ratio between the signal level induced in the antenna and the field intensity. The length of the protruding conductor must not exceed the desired spatial resolution. Unfortunately, the resolution does not just depend on this length, but also on the dimensions of the shielding as surface currents in the shielding induce a secondary field and change the input signal. When the field is highly localized around the apex of the protruding conductor, images with spatial resolution somewhat better than the length of the conductor and the dimensions of the shielding can be obtained. On the other hand, those images lack good quantitative characterization, as the antenna's signal level depends on a particular distribution of the field that can no longer be considered to be homogeneous. Additionally, when the antenna length is chosen to be comparable or shorter than the shield diameter $D$, the presence of the shielding close to the circuit may cause redistribution of the charges in the circuit and distortion of the primary induced field.

It appears that only by decreasing the antenna dimensions along with the coaxial shielding that its spatial resolution capability can be improved. Unfortunately, miniaturization of the 
antenna to the micrometer range makes its fabrication rather difficult, especially manufacturing the coaxial line of low diameter and the forming of a short protruding central conductor. Our measurement method, which we shall call the position/signal difference (PSD), overcomes the resolution limit determined by the antenna's dimensions and allows the increase of its resolution capability without the need for further miniaturization of the antenna. The method is based on comparing results of two subsequent scans with the antenna displaced by a small distance $\Delta l$ along its axis. For planar microwave circuits, the strength of the field is greatest close to the circuit surface and decays with increasing distance from the surface. For a thin short antenna $(d \ll l, l \ll \lambda / 4)$, placed in such a nonhomogeneous field, the signal level can be approximated as a sum of the field contributions acting along the protruding conductor. If we split the antenna interaction areas to three regions - the antenna apex $\mathcal{A}$, middle section of the antenna protruding conductor $\mathcal{B}$, and the input to the shield $\mathcal{C}$ - the resulting signals $I_{1}, I_{2}$ before and after antenna displacement can be formally written as

$$
\begin{aligned}
& I_{1}=I_{1}^{\mathcal{A}}+I_{1}^{\mathcal{B}}+I_{1}^{\mathcal{C}} \\
& I_{2}=I_{2}^{\mathcal{A}}+I_{2}^{\mathcal{B}}+I_{2}^{\mathcal{C}} .
\end{aligned}
$$

We describe the signal levels in terms of the induced currents $I_{1}, I_{2}$ at the input to the coaxial shielding, as the impedance of our short antennas are high in comparison with the input impedance of the subsequent network and, therefore, the antenna functions are more of a current source. Geometry of the protruding conductor in the middle region $\mathcal{B}$ does not change with the antenna displacement and the contribution from the same external field remains unchanged $\left(I_{1}^{\mathcal{B}}=I_{2}^{\mathcal{B}}\right)$. For highdensity structures, the field strength in the region $\mathcal{C}$ and its contribution to the overall signal can be assumed to be negligible $\left(I_{1}^{\mathcal{C}}=I_{2}^{\mathcal{C}}=0\right)$. The measured signal difference

$$
\Delta I=I_{2}-I_{1}=I_{2}^{\mathcal{A}}-I_{1}^{\mathcal{A}}
$$

depends only on the field surrounding the displaced antenna apex. In this way, the field surrounding the conductor apex can be isolated and measured and the spatial resolution of the microwave field mapping can be significantly improved.

Unlike the case for far-field imaging systems, for near-field measurements, there is no exact definition of the spatial resolution. This lack of definition is caused by the fact that the probe response is affected by the particular distribution of the external field surrounding the active region of the probe. For this reason, the response of the probe is usually determined by assuming that it is located in a homogeneous external field and its spatial resolution corresponds to the dimensions of the active region of the probe. In the case of the PSD method, the difference signal is determined by the changes in the boundary conditions at the apex of conductive protruding conductor during its displacement. These changes are limited to the region $\Delta l$, and the measured signal and resolution of the method are determined by the displacement $\Delta l$.

To demonstrate the effect of the resolution enhancement, we have measured the vertical $z$-component of the electric field above area $A$ of an open-ended transmission line forming a letter

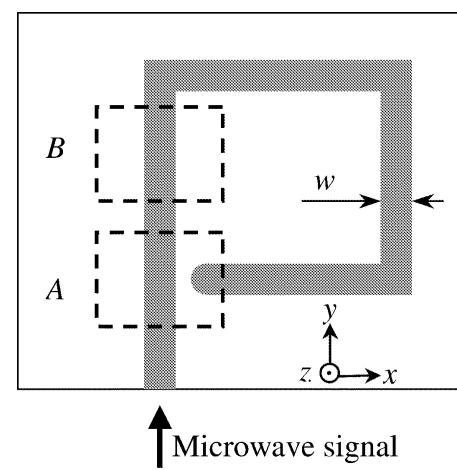

Fig. 2. Testing circuit: an open-terminated microstrip transmission line was prepared by a standard lithographic method on a microwave PCB board. The dielectric substrate has permittivity $\epsilon=6.2$ and thickness $t=510 \mu \mathrm{m}$, the widths of the lines are $w=250$.

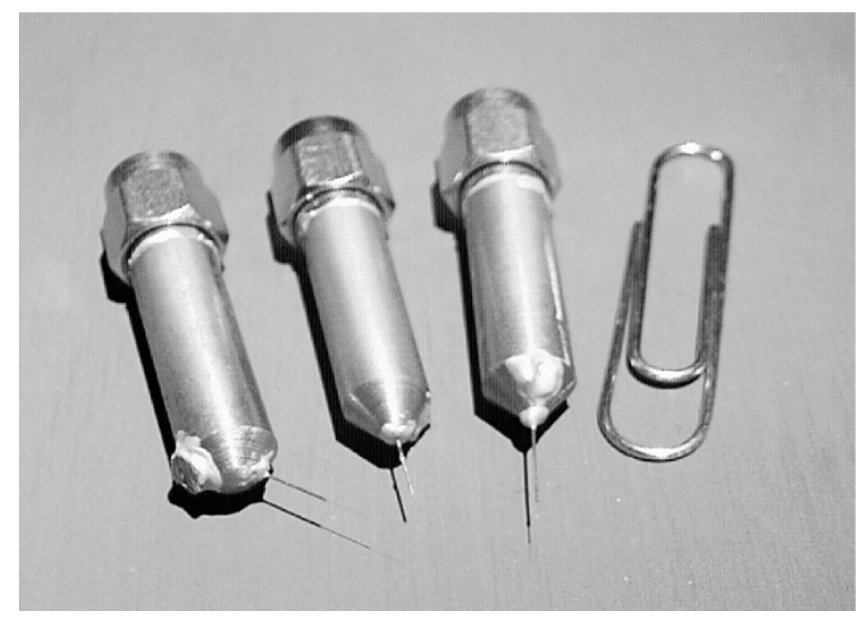

Fig. 3. Electric-field probes. The probe on the left-hand side is designed for measurement of normal and tangential spatial-field components. The probe incorporates a low-noise monolithic-microwave integrated-circuit (MMIC) Dahele preamplifier, and the output signal and dc bias are coupled through a standard subminiature A (SMA) connector. The connector also allows rotation of the antennas around the vertical axis.

"P" (see Fig. 2). The antennas (Fig. 3) are driven using precision motorized stages with specified separation $\Delta z$ above the circuit. Fig. 4(a) and (b) represents field images of the normal electric-field acquired for two different antenna/sample separations and (c) is the difference of these signals. We can clearly observe significant resolution enhancement for the difference signal. As the antenna is sensitive to the field acting along the entire length of the protruding conductor, the scattered field intensities at higher distances above the sample represent the main contribution to the level of the acquired signal. The difference signal corresponds to the local electric-field intensities surrounding the antenna apex only.

To determine the highest achievable resolution of our antennas, we have prepared a printed circuit board (PCB) surface capacitor with a small separation gap between its fingers (see Fig. 5). The antenna was driven very close to the sample surface according to previously acquired topographic data. The acquisition of the sample topography is necessary to keep the separation between the antenna and sample constant. Due to the sample tilt and step-like features of the sample surface, which may cause the variation of this separation or even exceed the 


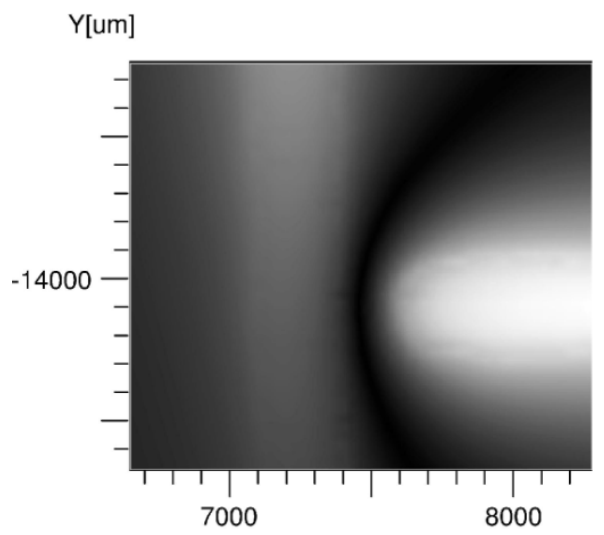

(a)

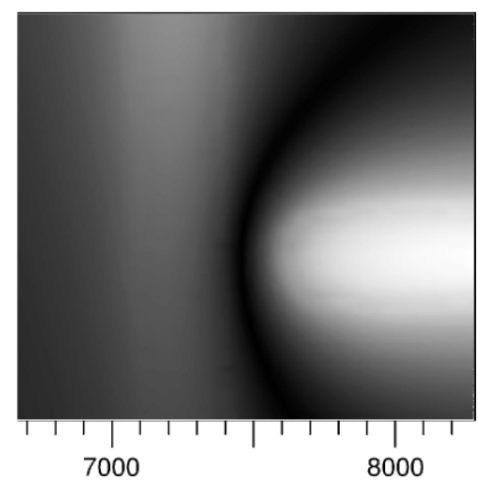

(b)

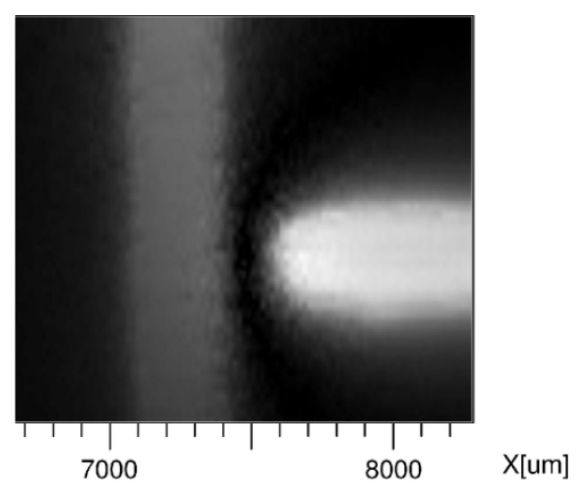

(c)

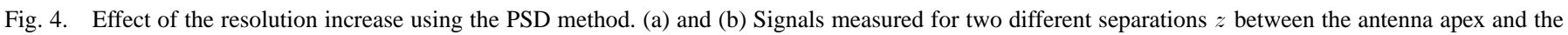

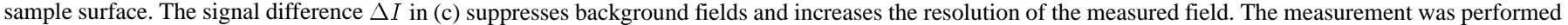
for a microwave signal of frequency $f=2 \mathrm{GHz}$.

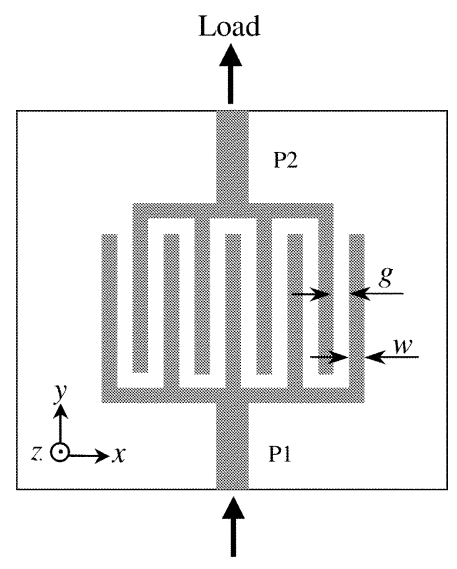

Microwave signal

(a)

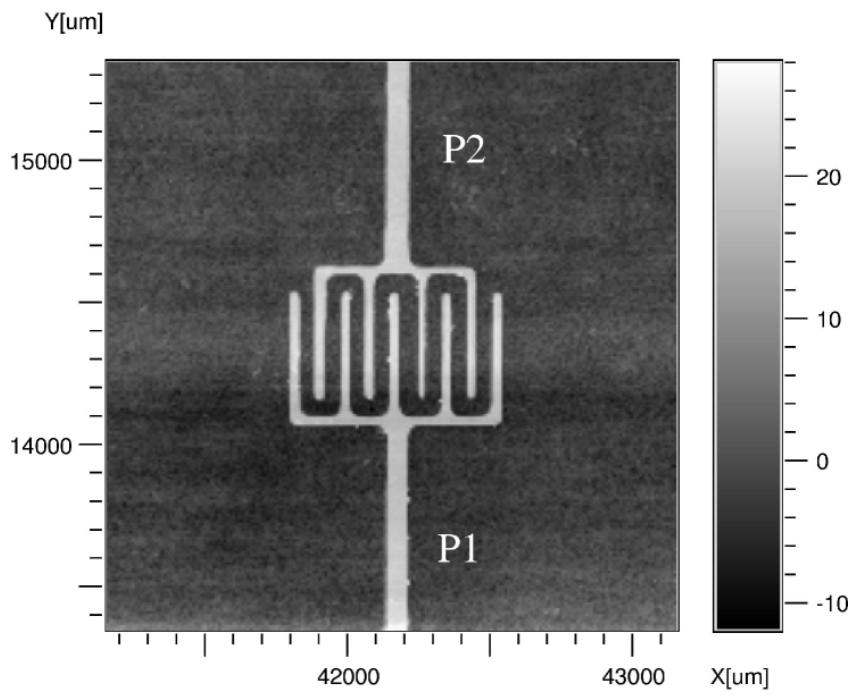

(b)

Fig. 5. PCB surface capacitor. The dielectric substrate has permittivity $\epsilon=$ 10.2 and thickness $t=127 \mu \mathrm{m}$. The widths of the fingers are $w=40 \mu \mathrm{m}$, and they are separated by a gap $g=60 \mu \mathrm{m}$. The topography of the circuit surface in (b) was imagined by a tuning-fork-based acquisition system. It is formed by a pattern of a copper layer with a thickness of approximately $t_{l}=17 \mu \mathrm{m} . P 1$ and $P 2$ indicate input and output ports, respectively.

working distance and cause the collision of the antenna with the sample surface, conventional horizontal plane scanning [1],
[2], [6] cannot be implemented. We have used an atomic force microscopy (AFM)-like technique utilizing a quartz tuning fork [7], [8] for acquisition of the sample topography, as presented in Fig. 5(b). After that, the topography probe is exchanged for the field antenna and field measurements are performed for various separations between the antenna's apex and surface. A static reference tip is used when the topography probe is exchanged for the microwave antenna, and the probes are aligned by means of an optical control using a long-focal length microscope.

The signal from the source of a vector network analyzer (VNA) is coupled to the input port $P 1$ of the capacitor. The output port $P 2$ is terminated by a short circuit at a distance of approximately $25 \mathrm{~mm}$ from the capacitor to allow excitation of the capacitor to higher potentials due to resonance in the transmission line at the frequency of interest $f=3.84 \mathrm{GHz}$. Fig. 6(a) and (b) is the microwave signals for antenna/sample separations of 5 and $12 \mu \mathrm{m}$, respectively, and (c) is the difference of these signals. The analysis of the field images would suggest that the spatial resolution obtained by the PSD method is approximately $20 \mu \mathrm{m}$, which gives the ratio of the resolution to the wavelength $R / \lambda$ of some $2 \cdot 10^{-4}$.

\section{ACQUiSITION OF TANGENTIAL COMPONENTS OF THE ELECTRIC FIELD}

Although in many cases measurement of the vertical electric intensity $E_{z}$ is the most appropriate method for the investigation of circuit signals, some situations may require examination of other spatial components of the field. Knowledge of the tangential-field components $E_{x}, E_{y}$ can be useful, i.e., for the description of fields of coplanar transmission lines and other structures with strong fields parallel to the circuit surface or for measurements of the field coupling between different parts of a device. The antennas for acquisition of normal and tangential components of the electric field are usually different: in most cases, for the latter, small dipoles are used [9], [10]. Simultaneous mapping of the various spatial-field components using an alternative approach with electrooptical detection was also reported [11].

The PSD method gives us an opportunity to measure all spatial components with a single probe. To accomplish this goal, we have modified the configuration of our probes by placing the coaxial antenna with an inclination of approximately $\alpha=45^{\circ}$ 


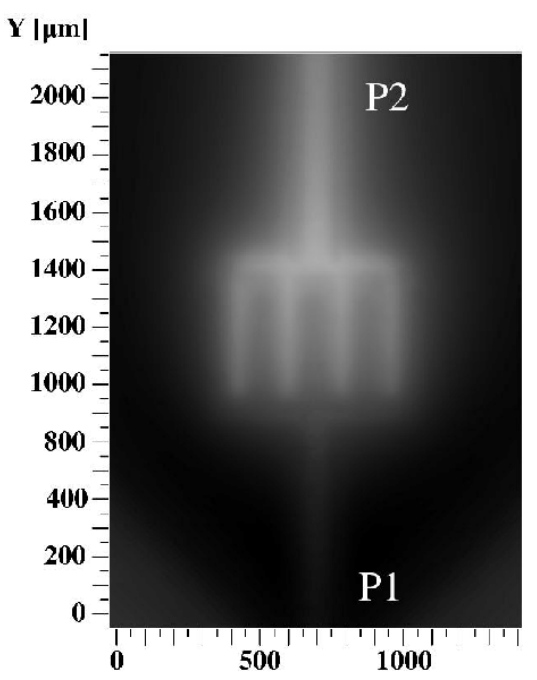

(a)

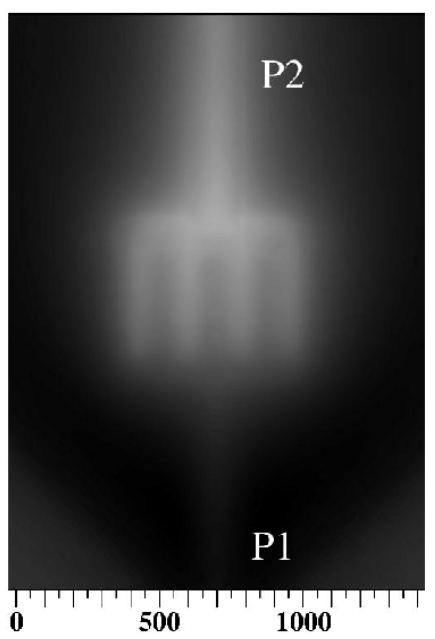

(b)

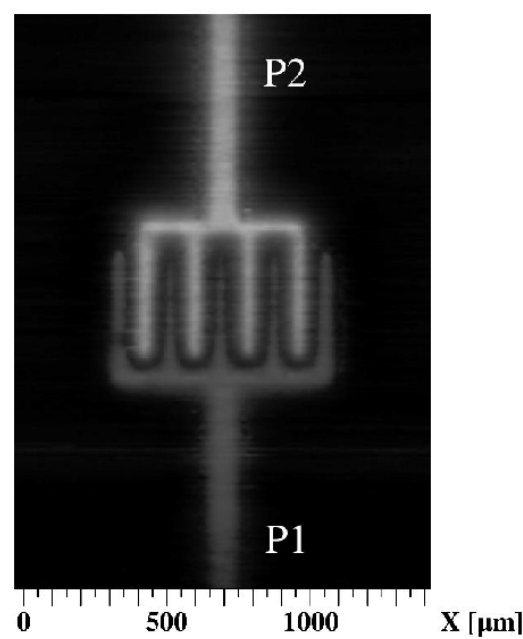

(c)

Fig. 6. (a) and (b) Signals acquired for antenna/sample separation of 5 and $12 \mu \mathrm{m}$, respectively. The difference signal (c) significantly increases spatial resolution of the field mapping. It also reveals weak local field intensities close to the signal lines of the input port $P 1$ of the capacitor, otherwise masked by strong background signals. These signals are induced by background fields acting along the whole length of the protruding conductor above the displaced apex of the antenna.

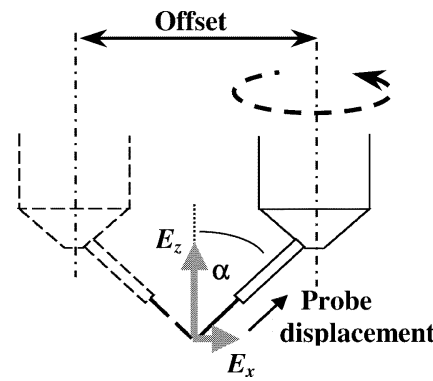

Fig. 7. Antenna configuration for measurement of normal and tangential components of the electric-field intensity. After each rotation, the antenna is aligned using a static reference tip so that its apex is placed at the same location. The offset, resulting from such an alignment, is then used during the scanning process for correct antenna placement.

relative to the vertical axis (see Fig. 7). By rotating such an antenna about that axis, different spatial components can be measured. Standard Cartesian intensities, perpendicular and parallel relative to the circuit surface plane, can be further recalculated. In the case of two measurements with the probe rotated by $180^{\circ}$ around the normal axis, a vertical and one tangential-field intensity can be obtained as follows:

$$
\begin{aligned}
& E_{z}=\frac{1}{2 \cos \alpha}\left(E_{0^{\circ}}+E_{180^{\circ}}\right) \\
& E_{t}=\frac{1}{2 \sin \alpha}\left(E_{0^{\circ}}-E_{180^{\circ}}\right) .
\end{aligned}
$$

Here, $E_{0^{\circ}}, E_{180^{\circ}}$ are the electric-field intensities detected by the antenna before and after the rotation. For three measurements with the antenna rotated by angles $0^{\circ}, 120^{\circ}$, and $240^{\circ}$, all three components can be calculated as follows:

$$
\begin{aligned}
& E_{x}=\frac{1}{\sin \alpha}\left(2 E_{0^{\circ}}-E_{120^{\circ}}-E_{240^{\circ}}\right) \\
& E_{y}=\frac{1}{\sqrt{3} \sin \alpha}\left(E_{120^{\circ}}-E_{240^{\circ}}\right) \\
& E_{z}=\frac{1}{3 \cos \alpha}\left(E_{0^{\circ}}+E_{120^{\circ}}+E_{240^{\circ}}\right) .
\end{aligned}
$$

In general, the field can be elliptically polarized and the phase of the electric intensities may vary for different spatial directions. Therefore, both the amplitude and phase of the signal must be acquired by a VNA, and the intensities of the electric fields in (3) and (4) represent complex amplitudes of the signal.

As the rotation about the vertical axis changes the probe's position above the sample surface, we must offset the probe position so that the displaced apex of the central protruding conductor is located at the same point before and after the antenna rotation. This position is adjusted using a reference tip following a procedure similar to the one used for alignment of the topography probe and the antenna for high-resolution measurements. It is also important to keep the rotation axis vertical so that the declination angle $\alpha$ remains constant for all antenna positions. A constant declination angle is crucial for calculation of the tangential electric field to remove the influence of the normal-field component. This is especially important for measurements of microstrip transmission lines, where normal-field components are typically several times stronger than tangential ones.

The tangential components in Fig. 8 were acquired using a $45^{\circ}$ inclined antenna. The measurements were performed above area $B$ of the microstrip (Fig. 2) with antennas aligned in two opposite directions perpendicular to the strip. In this experiment, the distance from the circuit surface was chosen to be larger $(600 \mu \mathrm{m})$, as the tangential components are negligible close to the circuit surface and vanish at the conductive boundaries of the strip or grounding. For each direction, two scans were performed with the antenna displaced along the inclined protruding conductor, their difference represents the field intensities at the antenna apex [see Fig. 8(a)]. Normal $E_{z}$ and tangential $E_{x}$ electric-field components [see Fig. 8(c)] were obtained using (3). As expected, the tangential component $E_{x}$ vanishes at the very center of the microstrip where the vector of electric intensity is perpendicular to the sample surface. Note that the tangential component does not vanish above the entire area of the strip line. At greater elevation above the surface of the transmission line, both normal and tangential-field components have 


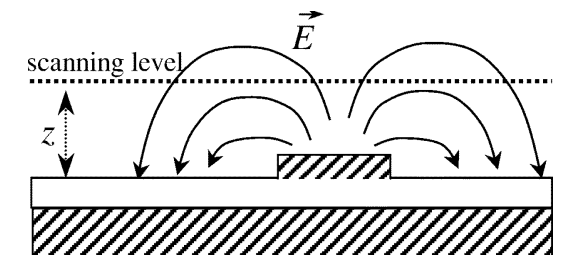

(a)

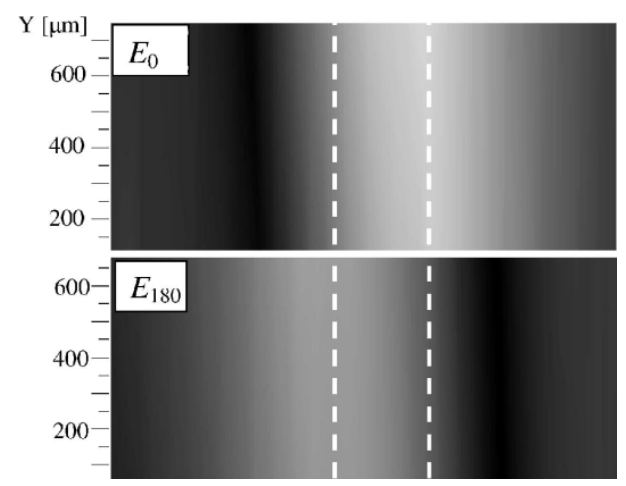

(b)

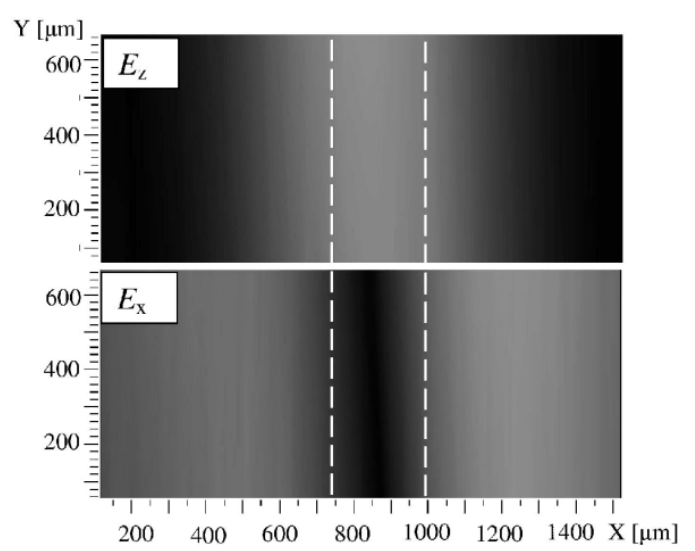

(c)

Fig. 8. Measurement of normal and tangential spatial components of the electric field for separation $z=600 \mu \mathrm{m}$ above area $B$ of the microstrip transmission line. Schematics (a) shows the distribution of the electric field across the line. In (b), the intensities for the inclined antenna were measured for two directions with the antenna rotated by $180^{\circ}$. For each direction, the PSD method was used to limit the measured electric-field intensity to the antenna apex. Normal $E_{z}$ and tangential $E_{x}$ field intensities in (c) were calculated according to (3) as a sum and difference of those signals. The position of the strip edges is highlighted by dashed lines.

more continuous distributions and the resolution of the field map is lower than the one for measurements presented in Figs. 4 and 6. For this field pattern, a reduction in the value of the antenna displacement $\Delta l$ in the PSD method would not result in further resolution increase. Therefore, the measurements were taken with a relatively large value $\Delta l=50 \mu \mathrm{m}$ to increase the level of the signal difference. We would like to stress that the reduced resolution in Fig. 8 is a reflection of the field distribution rather than of the capabilities of the PSD method.

\section{Sensitivity OF THE SyStem AND CALIBRATION OF THE PROBES}

The level of the acquired signal depends not only on the signal induced in the apex of the conductor, but also on the

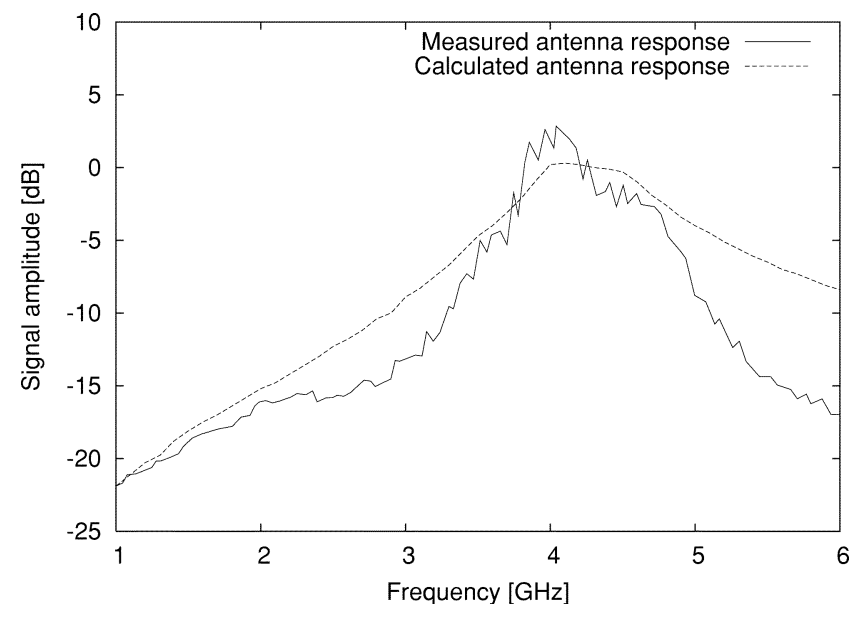

Fig. 9. Measured and calculated frequency response of the antenna. Real scattering parameters of the preamplifier were used for the calculations of the response. For low frequencies, we can expect much smaller variation in electrical properties of the antenna caused by uncertainties in antenna geometry and wave character of signal propagation. As the precision of calculated absolute sensitivity is limited by the accuracy of the antenna model used, both curves were fitted for values close to $1-\mathrm{GHz}$ frequency. The design of the antenna was optimized for higher sensitivity $S$ at 4-GHz frequency.

efficiency of its matching to the input of the coaxial line, the properties of the preamplifier, and the transmission of the signal to the acquisition system to the VNA. One of the main factors influencing the level of acquired signal is the impedance of the antenna. It is determined by its geometry, especially by the length and diameter of the protruding conductor. Although its precise value has to be determined by numerical calculations [12], [13], we can estimate it by using an approximation of Hallén's equations for thin short wire antennas $(l \ll \lambda / 4, d \ll l)$. For our short antennas $(0.3-1 \mathrm{~mm})$ and frequencies of interest (1-5 GHz), the impedance has a nearly pure capacitive character $\left(Z_{X} \approx-j Z_{0} \lambda \ln \left((l / d) /\left(4 \pi^{2} l\right)\right)\right)$ with values in the range of $10^{3}-10^{4} \Omega$. The protruding conductor functions as a nearly ideal current source and it is difficult to match its high impedance to the subsequent preamplifier with a standard impedance of approximately $Z_{A} \simeq 50 \Omega$. To improve the matching efficiency, the input impedance to the coaxial line - as seen by the active protruding conductor-has to be increased. For our miniature probes, we have chosen a simple matching scheme that uses a quarter-wavelength transformer formed by the antenna coaxial input line with a relatively high characteristic impedance of approximately $Z_{c} \simeq 120 \Omega$. By choosing the length of this line to be equal to $\lambda / 4$ for the frequency of interest $(4 \mathrm{GHz})$, the increase in the signal level resulting from this matching procedure should be approximately $12 \mathrm{~dB}$. An example of the antenna response is shown in Fig. 9. The antenna was placed in a well-defined field above an air-suspended cylindrical transmission line for which the surrounding field can be easily calculated. Although the measured data follow the general character of theoretical curves, they do not fully match at particular values. The difference is especially significant at high frequencies for which the response is much lower than expected from theoretical calculations. The difference is caused by an inaccuracy of the antenna model and also by tolerances in the antenna dimensions. 


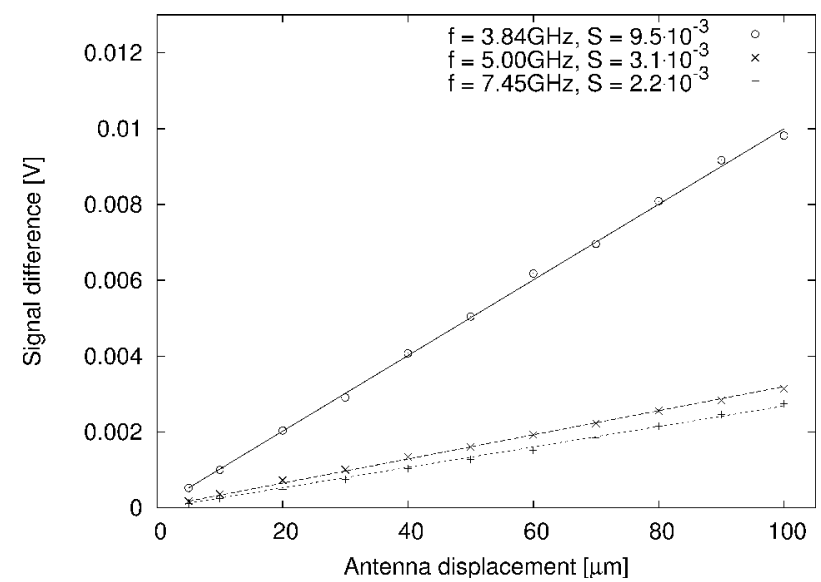

Fig. 10. Calibration of the antennas for the PSD method. We can observe highly linear dependency of the measured signal difference $\Delta V$ on antenna displacement $\Delta l$. The measurement was performed using a calibration unit-an air-suspended cylindrical transmission line for which the field at any coordinate can be calculated.

For short antennas, placed in a homogeneous field, the signal from the antenna is proportional to the length of the protruding conductor. For the PSD method, the displacement of the antenna corresponds to a virtual change in the length of its protruding conductor. Although the conductor is not placed in the homogeneous field, the antenna response signal can be regarded as the sum of the field contributions acting along the protruding conductor. As the field intensity in a small region around the displaced antenna apex is assumed to be constant, the signal difference should be proportional to the displacement $\Delta l$. The experimental results (see Fig. 10) confirm the linear character of this dependency. As a typical preamplifier is also highly linear, the measured voltage $\Delta V$, after its conditioning and transmission to the input of the acquisition instrument (i.e., VNA), is proportional to the antenna displacement $\Delta l$. We can, therefore, define the sensitivity of the system for a particular frequency by a single unitless constant $S$

$$
S=\frac{\Delta V}{E_{l} \Delta l}
$$

Here, $E_{l}$ is the component of the electric-field intensity of the microwave field parallel to the antenna axis. Due to the variation of the antenna electrical properties, each antenna has to be individually calibrated in a well-defined field standard, such as the above-mentioned cylindrical line. Other microwave circuits (i.e., microstrip transmission line) can be also used as long as the surrounding field can be explicitly calculated. The sensitivity constant $S$, determined from calibration measurements, can be used for the calculation of real values of the electric-field intensity during the scanning process.

The analysis of the PSD method shows that one can improve the resolution by reducing the displacement $\Delta l$. At the same time this leads to a reduction in the level of signal difference. As this difference must exceed the noise level, the sensitivity of the antenna may effectively limit its resolution and make it dependent on minimal detectable field intensities. The noise floor level $-95 \mathrm{dBm}$ for $10-\mathrm{Hz}$ bandwidth and measured sensi- tivity constant at 4-GHz frequency give us a minimum level of detectable electric-field intensity of approximately $10 \mathrm{~V} / \mathrm{m}$ for displacement of approximately $30 \mu \mathrm{m}$ and comparable spatial resolution.

\section{CONCLUSION}

Inspection of particular microwave elements and the description of the field surrounding them is especially important during the development and testing phase of microwave devices when maximum information about their functionality is desired. High spatial resolution and low distortion of the measured field are key considerations for the adoption of near-field measurements as a noninvasive technique for the testing of microwave devices. In order to measure microwave electric-field intensities with high spatial resolution, we have developed special miniaturized field probes and measurement techniques. In particular, the PSD method appears to be an effective approach to measure the electric-field intensity in the deep near-field region $\left(\lambda / 10^{4}\right)$ and allows one to achieve exceptional resolution with low distortion of the measured field and good quantitative field characterization. The method also makes it possible to use a single probe for the measurement of all spatial components of the electric field. The PSD technique works well only with high gradient fields. This is indeed the case for most high-density structures where the electric intensity decays rapidly with increasing distance above the circuit. However, for larger structures, where the field patterns are more homogeneous, there is no need for the use of high-resolution antennas. Standard measurement methods or the PSD method with a longer protruding conductor can be successfully used in such cases.

Our future research will focus on the evaluation of the method for various distribution of the external field and its comparison with a numerical model. Although our preliminary simulations using moment methods confirm the results presented in this paper, it appears that a full-wave solution (such as the finite-difference time-domain method) is required for a better description of the antenna properties and PSD method. We will also consider the modification of the technique utilizing a narrow loop antenna for the acquisition of the magnetic components of the field. Such measurements would give complementary information on the distribution of currents in the device-under-test.

\section{REFERENCES}

[1] J. S. Dahele and A. L. Cullen, "Electric probe measurements on microstrip," IEEE Trans. Microwave Theory Tech., vol. MTT-28, pp. 752-755, July 1980

[2] Y. Gao, A. Lauder, Q. Ren, and I. Wolf, "Calibration of electric coaxial near-field probes and applications," IEEE Trans. Microwave Theory Tech., vol. 46, pp. 1694-1703, Nov. 1998.

[3] T. Nozokido, J. Bae, and K. Mizuno, "Scanning near-field millimeterwave microscopy using a metal slit as a scanning probe," IEEE Trans. Microwave Theory Tech., vol. 49, pp. 491-499, Mar. 2001.

[4] M. Golosovsky, A. Lann, and D. Davidov, "A millimeter-wave near-field scanning probe with optical distance control," Ultramicroscopy, vol. 71, pp. 133-141, 1998.

[5] M. Fee, S. Chu, and T. W. Hansch, "Scanning electromagnetic transmission line microscope with sub-wavelength resolution," Opt. Commun., vol. 69, pp. 219-224, 1989. 
[6] S. K. Dutta, C. P. Vlahacos, D. E. Steinhauer, A. S. Thanawalla, B. J. Feenstra, F. C. Wellstood, and S. M. Anlage, "Imaging microwave electric fields using a near-field scanning microwave microscope," Appl. Phys. Lett., vol. 74, pp. 156-158, 1999.

[7] K. Karrai and R. D. Grober, "Piezoelectric tip-sample distance control for near-field optical microscopes," Appl. Phys. Lett., vol. 66, pp. 1842-1844, 1995.

[8] R. Kantor, M. Lesnak, N. Berdunov, and I. V. Shvets, "Method for increasing sensitivity of shear-force distance control for scanning nearfield microscopy," Appl. Surf. Sci., vol. 144-146, pp. 510-513, 1999.

[9] T. P. Budka, D. Waclawik, and G. M. Rebeitz, "Near electric field mapping above $X$-band MMICs using modulated scattering," in IEEE MTT-S Int. Microwave Symp. Dig., vol. 3, 1996, pp. 1703-1706.

[10] T. P. Budka, M. Tentzeris, D. Scott, D. Waclawik, N. I. Dib, L. P. B. Katehi, and G. M. Rebeitz, "Near-field mapping above coupled-line filter and MMIC," Microwave J., pp. 94-106, Mar. 1998.

[11] K. Yang, T. Marshall, M. Forman, J. Hubert, L. Mirth, Z. Popović, L. P. B. Katehi, and J. F. Whitaker, "Active-amplifier-array diagnostics using high-resolution electrooptic field mapping," IEEE Trans. Microwave Theory Tech., vol. 49, pp. 849-857, May 2001.

[12] R. Luebbers, L. Chen, T. Uno, and S. Adachi, "FDTD calculation of radiation patterns, impedance, and gain for a monopole antenna on a conducting box," IEEE Trans. Antennas Propagat., vol. 40, pp. 1577-1583, Dec. 1992.

[13] G. Liu and C. A. Grimes, "A quasi-static FDTD source model for coaxially driven monopole antennas," Microwave Opt. Technol. Lett., vol. 26 , pp. 30-34, 2000.

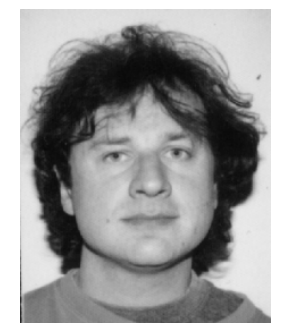

Roman Kantor was born in Ostrava, Czech Republic. He received the M.Sc. degree (with a specialization in optics and optoelectronics) from Palacky University, Olomouc, Czech Republic, in 1990. The subject of his research at the Technical University of Ostrava was ellipsometry and the study of optical properties of thin films. He received the $\mathrm{Ph} . \mathrm{D}$. degree in scanning near-field microscopy of microwave circuits from Trinity College, Dublin, Ireland, in 2001.

In 1997, he joined the Physics Department, Trinity College, Dublin, Ireland, where he is currently a Post-Doctoral Research Fellow.

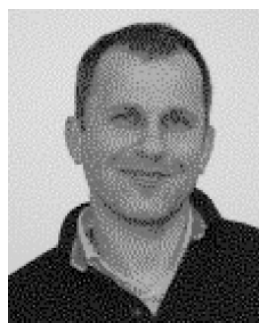

I. V. Shvets received the Ph.D. degree in magnetostriction in antiferromagnets, ferromagnets, and spin glasses from the Moscow Institute of Physics and Technology (MIPT), Moscow, Russia, in 1989.

Since 1991, he has been a Lecturer with the Physics Department, Trinity College, Dublin, Ireland. The laboratory for scanning probe techniques, which he established, actively participates in national and European research and development projects and collaborates with a number of universities and industrial companies.

Prof. Shvets has served as an expert and panel member for the European Commission in physics, nanotechnology, and related programs. 